\title{
Summarizing differences in cumulative incidence functions
}

\author{
Mei-Jie Zhang ${ }^{1,{ }^{*}, \dagger}$ and Jason Fine ${ }^{2}$ \\ ${ }^{1}$ Division of Biostatistics, Medical College of Wisconsin, Milwaukee, WI 53226, U.S.A. \\ ${ }^{2}$ Department of Biostatistics, University of North Carolina at Chapel Hill, Chapel Hill, NC 27599, \\ U.S.A.
}

\section{SUMMARY}

The cumulative incidence function is widely reported in competing risks studies, with group differences assessed by an extension of the log-rank test. However, simple, interpretable summaries of group differences are not available. An adaptation of the proportional hazards model to the cumulative incidence function is often employed, but the interpretation of the hazard ratio may be somewhat awkward, unlike the usual survival set-up. We propose nonparametric inferences for general summary measures, which may be time-varying, and for time-averaged versions of the measures. Theoretical justification is provided using counting process techniques. A real data example illustrates the practical utility of the methods.

\section{Keywords}

competing risk; counting processes; marginal probability function; nonparametric measures; timedependent summaries; two sample problem

\section{INTRODUCTION}

The cumulative incidence function quantifies the cause-specific failure probability with competing risks data, where the dependent censoring from competing causes invalidates the Kaplan-Meier [1] estimator. Nonparametric analyses of the cumulative incidence function have been widely adopted [2], particularly in clinical trial settings [3]. Counting process methods [4] may be used to nonparametrically estimate cause-specific hazard functions [5], which may then be combined to estimate the cumulative incidence function. The log-rank test has been extended [6] to evaluate group differences in cumulative incidence, with alternative testing procedures developed by Pepe [7] and Lin [8].

In two-group survival analyses with independent censoring, the Kaplan-Meier estimators are reported, along with results from the log-rank test and the hazard ratio estimated under the proportional hazards model [9]. With competing risks data, the nonparametric estimates of the cumulative incidence function are reported, frequently complemented by the results of Gray's [6] test. While the proportional hazards model has been adapted to the cumulative incidence function by Fine and Gray [10] and Scheike et al. [11], the interpretation of the corresponding hazard ratio is somewhat awkward and the analysis is often omitted. The estimated hazard ratios from the cause-specific hazard functions cannot be used to infer the magnitude of group differences on the cumulative incidence functions, directly.

Copyright $@ 2008$ John Wiley \& Sons, Ltd.

"Correspondence to: Mei-Jie Zhang, Division of Biostatistics, Medical College of Wisconsin, Milwaukee, WI 53226, U.S.A. .

$\dagger$ E-mail: meijie@mcw.edu 
In Section 2, we propose inferences for alternative summary measures based on nonparametric estimates of the cumulative incidence functions, which may be time-varying. This includes absolute difference, risk ratio, and odds ratio, which are more easily interpreted than the hazard ratio of the subdistribution proposed by Fine and Gray [10]. Theoretical issues are addressed using counting process techniques. The results permit the construction of confidence bands for time-varying summary measures and confidence intervals for time-averaged summary measures. The analyses give a fuller understanding of how group differences change over time than does the Fine and Gray [10] model.

The methodology's practical utility is illustrated in an analysis of a bone marrow transplant study in Section 3, where absolute differences and risk ratios are intuitively meaningful in quantifying covariate effects on the cumulative incidence functions. The case study shows that the effect of platelet levels on treatment related failure is strongest at early time points but wanes in the long run, which may be the time horizon of greatest clinical interest.

Some remarks conclude in Section 4.

\section{NONPARAMETRIC INFERENCES FOR SUMMARY MEASURES}

\subsection{Nonparametric estimation of cumulative incidence function}

We first review the usual nonparametric estimators for the cumulative incidence functions. Standard asymptotic results are presented, which are needed when making inference about the proposed summary measures. Simple plug-in formulas for the estimators and their associated variance estimators are given in the text, with additional details given in the Appendix.

For subject $j$ in group $i$, let $T_{i j}$ and $C_{i j}$ be the event time and censoring time, respectively. Let $X_{i j}=\min \left(T_{i j}, C_{i j}\right), \Delta_{i j}=\mathscr{I}\left(T_{i j} \leq C_{i j}\right)$, and $\varepsilon_{i j} \in\{1, \ldots, K\}$ be the cause of failure. For rightcensored competing risk data, we assume that for the $i$ th treatment group, $\left\{X_{i j}, \Delta_{i j}, \Delta_{i j} \varepsilon_{i j}\right\}$ are independent and identically distributed for $j=1, \ldots, n_{i}$, and assume that the observed data are independent between two treatment groups.

Let

$$
\lambda_{i}^{1}(t)=\lim _{\Delta t \rightarrow 0} \frac{\operatorname{pr}\left(t \leq T_{i j} \leq t+\Delta t, \varepsilon_{i j}=1 \mid T_{i j} \geq t\right)}{\Delta t}
$$

be the cause-specific hazard function of cause 1 in group $i=1,2$. The cumulative incidence function for cause 1 is

$$
F_{i 1}(t)=\operatorname{pr}\left(T_{i j} \leq t, \varepsilon_{i j}=1\right)=\int_{0}^{1} S_{i}\left(u^{-}\right) d \Lambda_{i}^{1}(u)
$$

where $S_{i}\left(u^{-}\right)=\operatorname{pr}\left(T_{i j} \geq u\right)$ is the overall survival probability and $\Lambda_{i}^{1}(u)=\int_{0}^{u} \lambda_{i}^{1}(v) \mathrm{d} v$ is the cumulative cause-specific hazard function of cause 1 .

To estimate the cumulative incidence function, $F_{i 1}(t)$, let $0=t_{i 0}<t_{i 1}<\cdots<t_{i D}$ be the distinct times where event of cause 1 occurred for the $i$ th sample. At time $t_{i j}$, let $Y_{i j}, r_{i j}$ and $d_{i j}$ be the number of subjects at risk, the number of events of cause 1 occurred and number of events of other than cause 1 occurred for the $i$ th sample. A nonparametric estimator of $F_{i 1}(t), i=1,2$, is given by 


$$
\widehat{F}_{i 1}(t)=\sum_{t_{i j} \leq t} \widehat{S}_{i}\left(t_{i, j-1}\right) d \widehat{\Lambda}_{i}^{1}\left(t_{i j}\right)
$$

where

$$
\widehat{S}_{i}(t)=\prod_{t_{i j} \leq t}\left\{1-\frac{r_{i j}+d_{i j}}{Y_{i j}}\right\} \quad \text { and } \quad \widehat{\Lambda}_{i}^{1}(t)=\sum_{t_{i j} \leq t} \frac{r_{i j}}{Y_{i j}}
$$

are the Kaplan-Meier estimator for the overall survival distribution, $S_{i}(t)$, and NelsonAalen [12,13] estimator for the cumulative hazard function, $\Lambda_{i}^{1}(t)$, respectively.

Andersen et al. [4] and Lin [8], among others, have studied the asymptotic properties of $\widehat{F}_{i 1}$. Under regularity conditions described in the Appendix, for $i=1,2$,

$$
n_{i}^{1 / 2}\left\{\widehat{F}_{i 1}(t)-F_{i 1}(t)\right\}=n_{i}^{-1 / 2} \sum_{j=1}^{n_{i}} I_{i j}^{F_{1}}(t)+o_{P}(1)
$$

uniformly in $t \in[0, \tau]$ for a fixed $\tau$, and $n_{i}^{1 / 2}\left\{\widehat{F}_{i 1}(t)-F_{i 1}(t)\right\}$ converges weakly in $D[0, \tau]$ to a tight Gaussian process. Moreover, $\operatorname{cov}\left\{\widehat{F}_{11}(s), \widehat{F}_{21}(t)\right\}=0$, for all $s, t$. The joint uniform asymptotic normality of the nonparametric estimators is important when developing inferences for summary measures.

For variance estimation, we require a consistent estimator of the influence function $I_{i j}^{F_{1}}(t)$, say, $\widehat{I}_{i j}^{F_{1}}(t)$. The influence function is complicated, so we omit the formulas here. Interested readers may find explicit expressions for $I_{i j}^{F_{1}}(t)$ and $\widehat{I}_{i j}^{F_{1}}(t)$ in the Appendix. A consistent variance estimator for $n_{i}^{1 / 2}\left\{\widehat{F}_{i 1}(t)-F_{i 1}(t)\right\}$ may be based on the estimated influence function:

$$
\widehat{\Sigma}_{i 1}^{F_{1}}(t)=\frac{1}{n_{i}} \sum_{j=1}^{n_{i}}\left\{\widetilde{I}_{i j}^{F_{1}}(t)\right\}^{2}
$$

\subsection{Summarizing differences in cumulative incidence functions}

To summarize differences in cumulative incidence, we consider general functionals $G\left\{\left(F_{11}(t), F_{21}(t)\right)\right\}$, where $G(u, v)$ maps $(D[0, \tau], D[0, \tau])$ to $\Re$, and has absolutely continuous partial derivatives in $u$ and $v$, and is bounded at $F_{i 1}(t)$, for $t \in[0, \tau]$ [4]. When $G\left\{F_{11}(t)\right.$, $\left.F_{21}(t)\right\}$ is constant in $t$, the cumulative incidence functions satisfy certain transformation models with time-independent covariate effects $[14,15]$ including the proportional subdistribution hazards model [10]. We suggest three intuitively meaningful transformations, $G_{1}(u, v)=u-v, G_{2}(u, v)=u / v$, for $v>0$, and $G_{3}(u, v)=\{u /(1-u)\} /\{v /(1-v)\}$, for $0<u, v<1$. The function $G_{1}$ is the differences ofthe two cumulative incidence probabilities, while $G_{2}$ and $G_{3}$ may be interpreted as the relative risk and the odds ratio of these probabilities, respectively, at a time $t \in[0, \tau]$. These quantities have been widely used for summarizing differences of probabilities with binary data. In the current setting, where the 
cause-specific failure probabilities change over time, these measures are completely unspecified, so that the differences may be time-dependent.

To combine information in $G\left\{F_{11}(t), F_{21}(t)\right\}$ across time, we propose weighted average summaries,

$$
\bar{G}=\int_{\tau_{l}}^{\tau_{u}} G\left\{F_{11}(t), F_{21}(t)\right\} \mathrm{d} W(t)
$$

where $W(t)>0$ is a deterministic weight function, $\int_{\tau_{l}}^{\tau_{u}} \mathrm{~d} W(t)=1$, and $\left[\tau l, \tau_{\mathrm{u}}\right] \in[0, \tau]$ is the time region of interest. If $G\left\{F_{11}(t), F_{21}(t)\right\}=\beta$ is time-independent, then the weighted average corresponds to the regression parameter in a transformation model [14,15]. For example, with $G(u, v)=\log \{-\log (1-u)\}-\log \{-\log (1-v)\}, \beta$ is the subdistribution hazard ratio [10].

However, in general, ${ }_{G}$ does not require that such a model holds. The weighted integral $\bar{G}$ may be interpreted as an average difference, average relative risk, or average odds ratio for $G_{k}, k=1,2,3$, respectively, which may be intuitively meaningful for researchers.

The weighting idea is similar in spirit to that of Fleming and Harrington [16], who proposed a class of weighted log-rank tests for censored survival data. Mimicking the weighted logrank tests, one might consider a class of weights based on the cumulative incidence function from pooling the two samples. This class of weights is rather flexible, and permits one to give greater weight either to early or late departures between two cumulative incidence curves, as well as weighting equally over time. An attractive feature of the uniform weight is that the summary measure may be interpreted as a simple weighted average.

\subsection{Estimation of $\mathrm{G}\left\{\mathrm{F}_{11}(\mathrm{t}), \mathrm{F}_{21}(\mathrm{t})\right\}$}

We estimate $G\left\{F_{11}(t), F_{21}(t)\right\}$ by $G\left\{\widehat{F}_{11}(t), \widehat{F}_{21}(t)\right\}$. The following paragraphs provide details of variance estimation.

The weak convergence of the estimator at a fixed $t$ and a corresponding variance estimator can be established using functional delta method. Some additional notation and assumptions are needed to state these results. Let $G^{(1)}(u, v)=\partial G(u, v) / \partial u$ and $G^{(2)}(u, v)=\partial G(u, v) / \partial v$. Define $n=n_{1}+n_{2}$ and assume that $n_{i} / n \rightarrow \rho_{i}$ as $n$, where $0<\rho_{i}<1$.

Since $\sqrt{n}\left\{\left(\widehat{F}_{11}(t), \widehat{F}_{21}(t)\right)-\left(F_{11}(t), F_{21}(t)\right)\right\}$ converges weakly to a Gaussian vector process, the functional delta method gives that $n^{1 / 2}\left[G\left\{\widehat{F}_{11}(t), \widehat{F}_{21}(t)\right\}-G\left\{F_{11}(t), F_{21}(t)\right\}\right]$ is uniformly asymptotically equivalent to

$$
\sum_{i} \sum_{j} \rho_{i}^{-1 / 2} G^{(i)}\left\{F_{11}(t), F_{21}(t)\right\} n_{i}^{-1 / 2} I_{i j}^{F_{1}}(t)=n^{1 / 2} \sum_{i} \sum_{j} I_{i j}^{G}(t)
$$

where the influence function $I_{i j}^{G}(t)$ can be estimated by $\widehat{I}_{i j}^{G}(t)=G^{(i)}\left\{\widehat{F}_{11}(t), \widehat{F}_{21}(t)\right\} \widehat{I}_{i j}^{F_{1}}(t) / n_{i}$ (see Appendix for detail). Thus, $n^{1 / 2}\left[G\left\{\widehat{F}_{11}(t), \widehat{F}_{21}(t)\right\}-G\left\{F_{11}(t), F_{21}(t)\right\}\right]$ converges weakly to a Gaussian process with variance consistently estimated by

$$
\widehat{\Sigma}_{G}(t)=n \sum_{i=1}^{2} \sum_{j=1}^{n_{i}}\left\{\widehat{I}_{i j}^{G}(t)\right\}^{2}=n \sum_{i=1}^{2} \frac{1}{n_{i}}\left[G^{(i)}\left\{\widehat{F}_{11}(t), \widehat{F}_{21}(t)\right\}\right]^{2} \widehat{\Sigma}_{i 1}^{F_{1}}(t)
$$




\subsection{Confidence intervals and bands for $G\left\{F_{11}(t), F_{21}(t)\right\}$}

For inferences about time-varying group differences, one may construct a $(1-\alpha) \times 100$ per cent confidence interval for $G\left\{F_{11}(\mathrm{t}), F_{21}(\mathrm{t})\right\}$. The interval endpoints may be calculated as $G\left\{\widehat{F}_{11}(t), \widehat{F}_{21}(t)\right\} \pm Z_{\alpha / 2}\left\{\widehat{\Sigma}_{G}(t) / n\right\}^{1 / 2}$, where $\operatorname{pr}\left(\mathrm{N}(0,1) \geq Z_{\alpha / 2}\right)=\alpha / 2$. For a fixed time $t \in[0, \tau]$, a pointwise hypothesis test of $H_{0}: F_{11}(t)=F_{21}(t)$ can be based on the asymptotic normality. For the difference of two cumulative incidence curves using $G_{1}$, a $(1-\alpha) \times 100$ per cent confidence interval for $\left\{F_{11}(t)-F_{21}(t)\right\}$ is

$$
\left\{\widehat{F}_{11}(t)-\widehat{F}_{21}(t)\right\} \pm Z_{\alpha / 2}\left\{\widehat{\Sigma}_{11}^{F_{1}}(t) / n_{1}+\widehat{\Sigma}_{21}^{F_{1}}(t) / n_{2}\right\}^{1 / 2}
$$

To construct the confidence interval for the relative risk and odds ratio with $G_{2}$ and $G_{3}$, respectively, we suggest an additional transformation, $f(G)$, to force the lower bound of the confidence interval to be positive and to improve the coverage probability in small samples. The log- and arcsine-square root transformations are well-known choices for $f$ [17]. For the log-transformation, the variance of $n^{1 / 2}\left\{\log \left[G\left\{\widehat{F}_{11}(t), \widehat{F}_{21}(t)\right\}\right]-\log \left[G\left\{F_{11}(t), F_{21}(t)\right\}\right]\right\}$ can be estimated by $\widehat{\Sigma}_{\log (G)}=\widehat{\Sigma}_{G}(t) /\left[G\left\{\widehat{F}_{11}(t), \widehat{F}_{21}(t)\right\}\right]^{2}$. Then, a $(1-\alpha) \times 100$ per cent logtransformed confidence interval for $G\left\{F_{11}(t), F_{21}(t)\right\}$ can be constructed as

$$
\exp \left[\log \left\{G\left\{\widehat{F}_{11}(t), \widehat{F}_{21}(t)\right\}\right\} \pm Z_{\alpha / 2} \frac{\sqrt{\widehat{\Sigma}_{G}(t) / n}}{G\left\{\widehat{F}_{11}(t) \widehat{F}_{21}(t)\right\}}\right]
$$

Computing (1- $\alpha) \times 100$ per cent confidence bands for $G\left\{F_{11}(t), F_{21}(t)\right\}$ may be helpful in identifying those time regions where $F_{11}$ and $F_{21}$ are different. It is difficult to construct such bands analytically, since the estimated functional does not have a simple martingale structure. We adapt Lin's [8] resampling technique [18] to generate the confidence bands for the difference. For a general transformation function $G$, we construct $(1-\alpha) \times 100$ per cent confidence bands for $G\left\{F_{11}(t), F_{21}(t)\right\}$ by a simple resampling technique.

Let $Z_{i j}^{b} ; i=1,2 ; j=1, \ldots, n_{i} ; b=1, \ldots, N$ be independent standard normal variates. It can be shown that the simulated process

$$
\widehat{J}^{(b)}(t)=n^{1 / 2} \sum_{i} \sum_{j} n_{i}^{-1} G^{(i)}\left\{\widehat{F}_{11}(t), \widehat{F}_{21}(t)\right\} \widehat{I}_{i j}^{F_{1}}(t) Z_{i j}^{b}
$$

has the same limiting process as $n^{1 / 2}\left[G\left\{\widehat{F}_{11}(t), \widehat{F}_{21}(t)\right\}-G\left\{F_{11}(t), F_{21}(t)\right\}\right]$, uniformly for $t \in[0, \tau]$. Let $C_{\alpha}$ be the (1- $\left.\alpha\right) 100$ th percentile of

$$
\bar{J}^{(b)}=\sup _{t \in\left[\tau_{l}, \tau_{u}\right]}\left|\widehat{J}^{(b)}(t)\right|
$$

for $b=1, \ldots, N$. Then, $(1-\alpha) \times 100$ per cent confidence bands for $G\left\{F_{11}(t), F_{21}(t)\right\}$ are

$$
G\left\{\widehat{F}_{11}(t), \widehat{F}_{21}(t)\right\} \pm C_{\alpha}\left\{\widehat{\Sigma}_{G}(t) / n\right\}^{1 / 2}
$$


For the relative risk and odds ratio, a log- or arcsine-square root transformation may be used to construct the bands as well.

\subsection{Inference for time-averaged differences}

A natural estimator for the time-averaged difference, $\underset{G}{-}$, is

$$
\widehat{\bar{G}}=\int_{\tau_{l}}^{\tau_{u}} G\left\{\widehat{F}_{11}(t), \widehat{F}_{21}(t)\right\} \mathrm{d} W(t)
$$

The functional delta method gives that

$$
\begin{aligned}
n^{1 / 2}\{\widehat{\bar{G}}-\bar{G}\} & =\sqrt{n} \int_{\tau_{l}}^{\tau_{u}}\left[G\left\{\widehat{F}_{11}(t), \widehat{F}_{21}(t)\right\}-G\left\{F_{11}(t), F_{21}(t)\right\}\right] \mathrm{d} W(t) \\
& =n^{1 / 2} \sum_{i} \sum_{j} \widehat{\bar{I}} \overline{i j}+o_{P}(1)
\end{aligned}
$$

where $\widehat{I_{i j}^{G}}=\int_{\tau_{l}}^{\tau_{u}} \widehat{I}_{i j}^{G}(t) \mathrm{d} W(t)$. The Lindeberg-Feller central limit theorem yields that $n^{1 / 2}\{\widehat{\bar{G}}-\bar{G}\}$ converges in distribution to a normal random variable. The asymptotic variance can be estimated by $\widehat{\Sigma}_{\bar{G}}=n \sum_{i} \sum_{j}\left\{\widehat{I}_{i j}^{-}\right\}^{2}$. To make inference, normal theory $(1-\alpha) \times$ 100 per cent confidence intervals may be calculated for $\bar{G}$.

\section{BONE MARROW TRANSPLANT DATA}

We illustrate the proposed summary measures on data from the International Bone Marrow Transplant Registry, consisting of 408 patients treated with HLA-identical sibling bone marrow transplantation for myelodysplasia and having complete platelet data. Two competing endpoints are of interest [19]: treatment-related mortality (TRM) $[N=161$ (40 per cent)], defined as death in remission, and relapse $[N=87(21$ per cent $)]$. One hundred and sixty (39 per cent) patients were censored at end of study. A key issue in patient management is identifying factors that influence the cumulative incidence of these outcomes.

Fitting univariate proportional cause-specific hazards models provided risk factors associated with the cause-specific hazards for TRM and relapse, respectively. These include pre-transplantation platelet level $\left(>100 \times 10^{9} / \mathrm{L}[N=128(31\right.$ per cent $)]$ versus $\leq 100 \times 10^{9}$ / $\mathrm{L}[N=280(69$ per cent)]). The estimated cause-specific hazard ratios ( 95 per cent confidence intervals) are $0.55(0.38-0.79)$ and $0.82(0.52-1.28)$ for TRM and relapse, respectively. These results suggest higher platelet levels are strongly associated with a reduction in TRM and that there is a smaller, but insignificant, reduction in relapse. While useful, such analyses do not yield direct information about the magnitude of the platelet effect on the cumulative incidence of the endpoints.

The estimated subdistribution hazard ratios (95 per cent confidence intervals) 10 and $p$ values from Gray's [6] test are 0.59 (0.41-0.84) 0.003 and 1.03 (0.66-1.61) 0.880 for TRM and relapse, respectively. The results for TRM are quite similar from the two analyses, in part because of the relatively weak effect of platelets on the cause-specific hazard of relapse. Conversely, the platelet effect is weaker on the cumulative incidence of relapse, in part 
because of the relatively strong platelet effect on the cause-specific hazard of TRM. Intuitively, patients with high platelet levels experience TRM less frequently and hence have greater cumulative risk of relapse, attenuating the positive effect of high platelet levels on the cause-specific hazard for relapse.

The proposed methodology is now employed to elucidate the time-varying nature of the platelet effect. Figures 1 and 2 plot the cumulative incidence curves for TRM and relapse for the two platelet groups, along with the time-varying difference, relative risk, and odds ratio, respectively. They also show the 95 per cent confidence intervals and confidence bands from $N=1000$ resamples. These figures indicate that platelets have a time-varying effect on the cumulative incidence of TRM (see Figure 1), but not on relapse (see Figure 2). To formally test for time-varying effects, we fit the Fine and Gray's [10] proportional subdistribution hazards model with a time-dependent covariate. The test gives $p$-values of 0.05 and 0.92 for TRM and relapse, respectively, suggesting that the proportional subdistribution hazards model may not be a good fit for the cumulative incidence of TRM.

For TRM, one observes that patients with platelets $>100 \times 10^{9} / \mathrm{L}$ have a lower cumulative incidence, but the advantage diminishes over time (Figure 1). The confidence bands demonstrate that the differences are statistically significant for the first 36 months posttransplantation, but not at later times. Taking $W=1 /\left(\tau_{u}-\tau_{l}\right)$ in $\bar{G}$, where $\left(\tau_{l}, \tau_{u}\right)=(0.164,70)$ month, the average difference, relative risk, and odds ratio for platelets $>100 \times 10^{9} / \mathrm{L}$ versus $\leq 100 \times 10^{9} / \mathrm{L}$ are -14 per cent $(-24$ to -5 per cent; $p=0.003), 0.65(0.48-0.88 ; p=0.006)$, and $0.53(0.34-0.82 ; p=0.004)$, respectively, with the $p$-values being quite similar to that from Gray's test. The average summary measures provide important information about the magnitude of the cumulative incidence differences, and may be more easily interpreted than the subdistribution hazard ratio [10]. Patients with platelets $>100 \times 10^{9} / \mathrm{L}$ have a timeaveraged 14 per cent reduction in the incidence of treatment-related death, while the timeaveraged relative risk and odds ratio of treatment-related death are 65 and 53 per cent, respectively.

For relapse, patients with high or low platelets have almost identical cumulative incidence curves (Figure 2) that are not significantly different at any time point using the confidence band procedure. The average difference, relative risk, and odds ratio of relapse for platelets $>100 \times 10^{9} / \mathrm{L}$ versus $\leq 100 \times 10^{9} / \mathrm{L}$ are 1 per cent $(-7-9$ per cent; $p=0.853), 1.01(0.68-1.50$; $p=0.974)$, and $1.02(0.62-1.70 ; p=0.931)$, respectively. The $p$-values are similar to that from Gray's 6 test. These findings are helpful in confirming the earlier analyses, which could have potentially missed a subtle time-varying effect.

The case study has demonstrated that the proposed time-varying and time-averaged measures can be usefully applied to compare two cumulative incidence curves and provide important information about the magnitude of the differences. When goodness-of-fit tests indicate that time-dependent effects in cumulative incidence functions exist, reporting the estimated hazard ratio from the Fine and Gray [10] model may be misleading, since the model assumes that this ratio does not change over time. The definition of the time-averaged measure accounts explicitly for such time-dependence and permits analyses of quantities like risk ratio and odds ratio which may be more easily understood by practitioners.

The confidence band methodology was helpful for answering the question: 'At what times are the two cumulative incidence curves different?'. This may be particularly important in clinical applications, both for physicians and patients. For example, in the bone marrow transplant study, the effect of platelets only manifests in the short run, with long-run probabilities of treatment-related mortality not appearing to depend as strongly on platelet 
levels. If long-term treatment failure is of ultimate interest, then these long-run probabilities may be of greater clinical relevance in patient management.

\section{REMARKS}

In this paper, we proposed a general class of time-dependent summary measures for group differences in two cumulative incidence functions. For scenarios where the differences may vary over time, we suggested averaging the measures across time. In the case that the difference is time-independent, there is a simple relationship between the summary measure and a transformation model in which there is a single binary covariate coding the group effect [14]. Estimation methods for such regression models could be used, including [14,15]. However, the proposed estimators in the current paper have a simple closed form as well as simple plug-in variance estimators, leading to straightforward inferences. The existing regression methods are more complex computationally, requiring the solution of certain estimating equations. Moreover, their interpretation may be unclear under time-varying dependence, unlike our approach, in which the time-averaged summary measure is estimated explicitly. The ad hoc pseudo-value approach of [15] might also be employed to estimate the time-dependent summary measures at fixed times. However, the theoretical properties of the procedure are not fully understood and the construction of the pseudo-values seems computationally more intensive in light of the current proposal. Additional research is needed to rigorously explore this method in the present setting.

In the bone marrow transplant example, the effects of the covariates on the cause-specific hazard and cumulative incidence functions were in the same direction but with somewhat different magnitudes. As discussed in Section 3, this occurs because the effect of a covariate on the cumulative incidence of a particular cause is mediated via its direct effect on the cause-specific hazard of that cause and via its indirect effect on the cause-specific hazards for other causes. When the effects of a covariate on the cause-specific hazards are in opposite directions, it may occur that the covariate effect on the cumulative incidence function of a particular cause is opposite to its effect on the corresponding cause-specific hazard. In such cases, care is needed in interpreting the results, which requires examining the effects on all causes simultaneously. The proposed methods are useful in understanding time-varying differences in the cumulative incidence function, which may help to refine the evaluation of these covariate effects. Future research is planned to explore the manner in which covariate effects on the cause-specific hazard functions contribute to time-varying differences on the cumulative incidence functions.

FORTRAN code implementing the analysis is available from the authors upon request. $\mathrm{R}$ and SAS code is currently under development for future release.

\section{Acknowledgments}

J. F. was supported by NCI grant 1 R01 CA94893-01 and M. J. Z. was supported by NCI grant 2 R01 CA54706-10.

Contract/grant sponsor: NCI; contract/grant numbers: R01 CA94893-01, R01 CA54706-10

\section{APPENDIX}

Nonparametric estimation of $F_{i 1}, i=1,2$, may be based on counting process techniques (see Andersen et al. [4]). Let $N_{i j}(t)=\mathscr{I}\left(X_{i j} \leq t, \Delta_{i j}=1\right)$ and $N_{i j}^{1}(t)=\mathscr{S}\left(X_{i j} \leq t, \Delta_{i j} \epsilon_{i j}=1\right)$ be the counting process of all causes of failure and observed counting process of type 1 failure for the $j$ th individual in $i$ th group, respectively. Denote $N_{i}(t)=\sum_{j=1}^{n_{i}} N_{i j}(t)$ and $N_{i}^{1}(t)=\sum_{j=1}^{n_{i}} N_{i j}^{1}(t)$, and define the 
risk set as $Y_{i}(t)=\sum_{j=1}^{n_{i}} Y_{i j}(t)$, where $Y_{i j}(t)=\mathscr{I}\left(X_{i j} \leq t\right)$. Under regularity conditions, it is well known that

$$
\begin{aligned}
\sqrt{n_{i}}\left\{\widehat{S}_{i}(t)-S_{i}(t)\right\} & =-n_{i}^{-1 / 2} S_{i}(t) \int_{0}^{t} \frac{\mathrm{d} M_{i}(u)}{y_{i}(u)}+o_{P}(1) \\
\sqrt{n_{i}}\left\{\widehat{\Lambda}_{i}^{1}(t)-\Lambda_{i}^{1}(t)\right\} & =n_{i}^{-1 / 2} \int_{0}^{t} \frac{\mathrm{d} M_{i}^{1}(u)}{y_{i}(u)}+o_{P}(1)
\end{aligned}
$$

uniformly in $t \in[0, \tau]$, where $M_{i j}(t)=N_{i j}(t)-\int_{0}^{t} Y_{i j}(u) \mathrm{d} \Lambda_{i}(u)$ and $M_{i j}^{1}(t)=N_{i j}^{1}(t)-\int_{0}^{t} Y_{i j}(u) \mathrm{d} \Lambda_{i}^{1}(u)$ are the martingales corresponding to $N_{i j}(t)$ and $N_{i j}^{1}(t)$, respectively, and $\Lambda_{\mathrm{i}}(t)=-\mathrm{d}\left\{\log \left[S_{\mathrm{i}}(t)\right]\right\}$ is the cumulative hazard function for all causes in group $i$, and $y_{i}(u)=\lim _{n_{i} \rightarrow \infty} n_{i}^{-1} Y_{i}(u)$ (see Pepe [7], p. 772 for details). Thus, it follows that

$$
\sqrt{n_{i}}\left\{\widehat{F}_{i 1}(t)-F_{i 1}(t)\right\}=n_{i}^{-1 / 2} \sum_{j=1}^{n_{i}} I_{i j}^{F_{1}}(t)+o_{P}(1)
$$

uniformly in $t \in[0, \tau]$, where

$$
I_{i j}^{F_{1}}(t)=\int_{0}^{t} S_{i}\left(u^{-}\right) \frac{\mathrm{d} M_{i j}^{1}(u)}{y_{i}(u)}-\int_{0}^{t} S_{i}\left(u^{-}\right)\left\{\int_{0}^{u^{-}} \frac{\mathrm{d} M_{i j}(v)}{y_{i}(v)}\right\} \mathrm{d} \Lambda_{i}^{1}(u)
$$

which can be consistently estimated by a plug-in estimator [20],

$$
\widehat{I}_{i j}^{F_{1}}(t)=\int_{0}^{t} \frac{\widehat{S}_{i}\left(u^{-}\right)}{n_{i}^{-1} Y_{i}(u)} \mathrm{d} \widehat{M}_{i j}^{1}(u)-\int_{0}^{t} \widehat{S}_{i}\left(u^{-}\right)\left(\int_{0}^{u^{-}} \frac{\mathrm{d} \widehat{M}_{i j}(v)}{n_{i}^{-1} Y_{i}(v)}\right) \mathrm{d} \widehat{\Lambda}_{i}^{1}(u)
$$

where $\mathrm{d} \widehat{M}_{i j}^{1}(u)=\mathrm{d} N_{i j}^{1}(u)-Y_{i j}(u) \mathrm{d} \widehat{\Lambda}_{i}^{1}(u), \mathrm{d} \widehat{M}_{i j}(u)=\mathrm{d} N_{i j}(u)-Y_{i j}(u) \mathrm{d} \widehat{\Lambda}_{i}(u)$, and $\widehat{\Lambda}_{i}(u)=\int_{0}^{u}\left\{Y_{i}(s)\right\}^{-1} \mathrm{~d} N_{i}(s)$.

Note that $\widehat{F}_{i 1}(t) \stackrel{\mathscr{P}}{\longrightarrow} F_{i 1}(t)$ uniformly for $t \in[0, \tau]$ and $n_{i} / n \rightarrow \rho_{i}$ as $n \rightarrow \infty$, where $0<\rho_{i}<1$. Thus, by Taylor expansion,

$$
\sqrt{n}\left[G\left\{\widehat{F}_{11}(t), \widehat{F}_{21}(t)\right\}-G\left\{F_{11}(t), F_{21}(t)\right\}\right]=\sum_{i, j} \rho_{i}^{-1 / 2} G^{(i)}\left\{F_{11}(t), F_{21}(t)\right\} n_{i}^{-1 / 2} I_{i j}^{F_{1}}(t)+o_{P}(1)
$$

uniformly in $t \in[0, \tau]$.

\section{REFERENCES}

1. Kaplan EL, Meier P. Nonparametric estimation from incomplete observations. Journal of the American Statistical Association. 1958; 53:457-481.

2. Kalbfleisch, JD.; Prentice, RL. The Statistical Analysis of Failure Time Data. Wiley; New York: 2002. 
3. Gooley TA, Leisenring W, Crowley J, Storer BE. Estimation of failure probabilities in the presence of competing risks: new representations of old estimators. Statistics in Medicine. 1999; 18:695-706. [PubMed: 10204198]

4. Andersen, PK.; Borgan, $\varnothing$.; Gill, RD.; Keiding, N. Statistical Models based on Counting Processes. Springer; New York: 1993.

5. Prentice RL, Kalbfleisch JD, Peterson AV Jr, Flournoy N, Farewell VT, Breslow NE. The analysis of failure times in the presence of competing risks. Biometrics. 1978; 34:541-554. [PubMed: 373811]

6. Gray RJ. A class of $K$-sample tests for comparing the cumulative incidence of a competing risk. Annals of Statistics. 1988; 16:1141-1154.

7. Pepe MS. Inference for events with dependent risks in multiple endpoint studies. Journal of the American Statistical Association. 1991; 86:770-778.

8. Lin DY. Non-parametric inference for cumulative incidence functions in competing risks studies. Statistics in Medicine. 1997; 16:901-910. [PubMed: 9160487]

9. Cox DR. Regression models and life tables (with Discussion). Journal of the Royal Statistical Society, Series B. 1972; 34:187-220.

10. Fine JP, Gray RJ. A proportional hazards model for the subdistribution of a competing risk. Journal of the American Statistical Association. 1999; 94:496-509.

11. Scheike TH, Zhang MJ, Gerds TA. Prediction cumulative incidence probability by direct binomial regression. Biometrika. 2008; 95:205-220.

12. Nelson W. Theory and applications of hazard plotting for censored failure data. Technometrics. 1972; 14:945-965.

13. Aalen OO. Nonparametric inference for a family of counting processes. Annals of Statistics. 1978; 6:701-726.

14. Fine JP. Regression modeling of competing crude failure probabilities. Biostatistics. 2001; 2(1): 85-97. [PubMed: 12933558]

15. Klein JP, Andersen PK. Regression modeling of competing risks data based on pseudovalues of the cumulative incidence function. Biometrics. 2005; 61:223-229. [PubMed: 15737097]

16. Fleming TR, Harrington DP. A class of hypothesis tests for one and two samples of censored survival data. Communications in Statistics. 1991; 10:763-794.

17. Borgan $\varnothing$, Liest $\phi 1 \mathrm{~K}$. A note on confidence intervals and bands for the survival curve based on transformations. Scandinavian Journal of Statistics. 1990; 17:35-41.

18. Lin DY, Fleming TR, Wei LJ. Confidence bands for survival curves under the proportional hazards model. Biometrika. 1994; 81:73-81.

19. Sierra J, Perez WS, Rozman C, Carreras E, Klein JP, Rizzo JD, Davies SD, Lazarus HM, Bredeson CN, Marks DI, Boogaerts MA, Goldman J, Champlin RE, Keating A, Weisdorf DJ, de Witte TM, Horowitz MH. Bone marrow transplantation from HLA-identical siblings as treatment for myelodysplasia. Blood. 2002; 100:1997-2004. [PubMed: 12200358]

20. Bickel, PJ.; Klaasen, CA.; Ritov, Y.; Wellner, JA. Efficient and Adaptive Estimation for Semiparametric Models. Springer; New York: 1998. 

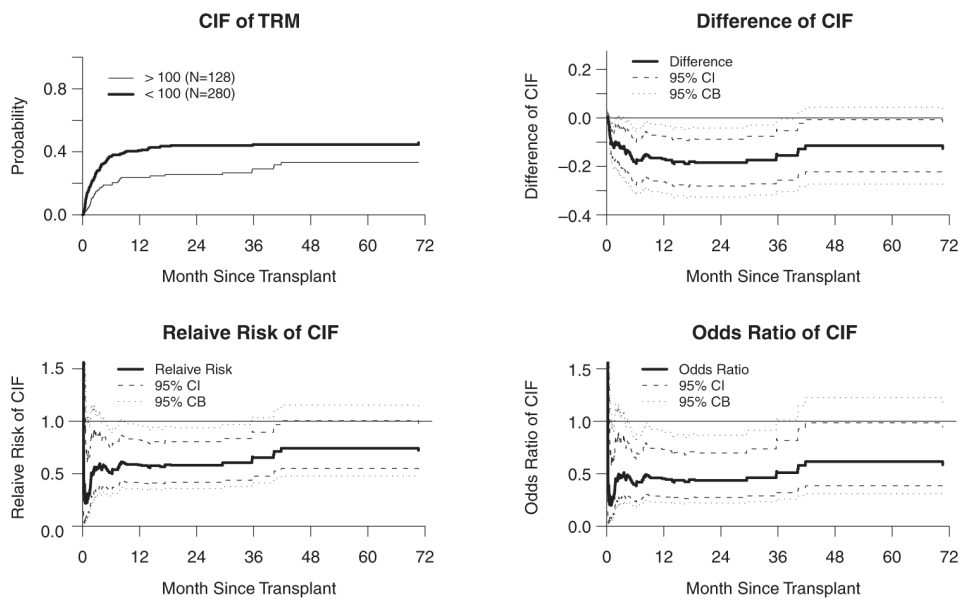

Figure 1.

Cumulative incidence function (CIF) of treatment-related mortality (TRM) by platelets (platelets $\left.>100 \times 10^{9} / \mathrm{L}\right)$ versus (platelets $\leq 100 \times 10^{9} / \mathrm{L}$ ). 

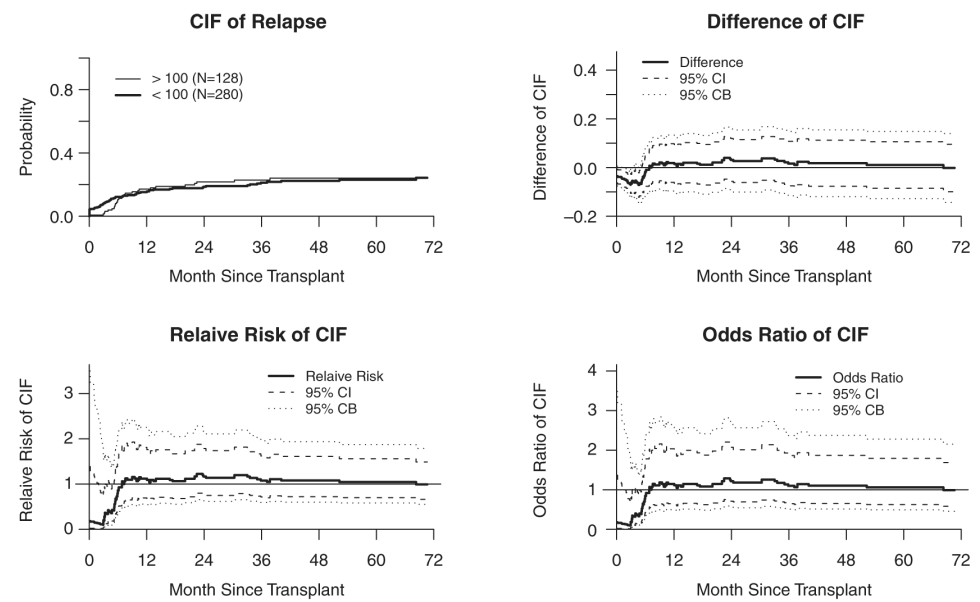

Figure 2.

Cumulative incidence function (CIF) of relapse by platelets (platelets $>100 \times 10^{9} / \mathrm{L}$ ) versus (platelets $\leq 100 \times 10^{9} / \mathrm{L}$ ). 